\title{
A RETROSPECTIVE ON THE USE OF SURVEY METHODOLOGIES IN MARKETING RESEARCH
}

\author{
David J. Ortinau, University of South Florida
}

\begin{abstract}
From a historical perspective, the methodologies used to collect and analyze various types of data structures in marketing research have undergone a number of popular trend changes from personal interviews and simple frequency, cross-tabulation, and correlation analysis procedures to complex experimental designs and advanced statistical analysis (ANOVA, MANOVA, SEM, and PLS).

Regarding survey methodologies, the advances in telecommunication technologies and methods (i.e., Internet and social media) of connecting with prospective respondents have significantly changed over the past two decades. For example, survey research during the 1970s and 1980s relied heavily on telephone and snail-mail, self-administered, pencil and paper survey methods as the two main methods of obtaining responses from a broad base of respondents. These methods required emphasis on scale measure designs, questionnaire layouts, as well as understanding defined target population characteristics in order to assure solid representation of a probability-based sample of respondents. Researchers' understanding of the different scale levels (i.e., nominal, ordinal, interval, ratio), scaling properties (i.e., assignment, order, distance, and origin), and the three basic elements that made up any scale measurement (i.e., the setup/question, desired attributes, and set of scale point descriptors) were critical in the designing of appropriate construct measures.
\end{abstract}

The 1990s and early 2000s ushered in the fast growing popularity of telecommunication technologies associated with the Internet and social media communities, influencing researchers' desires for faster, cheaper, electronic/online/email and web survey alternatives of collecting marketing research data. Well defined target populations and probability-based sampling designs were replaced with non-probability-based, convenience samples of respondents who either opt-in for participation or held membership in an identified social media community. Construct development and scale measurement procedures advocated in the 1980s, were rapidly replaced with "adopting" and/or "adapting" constructs and scales previously reported in the literature as means to save time, effort, and money.

The mobile telecommunication technologies of the late 2000s and 2010s (i.e., smart phones and Tablets) bring more advances and changes to survey research methods. In contrast, these new electronic advances create new data quality and data representativeness questions worthy of discussion.

This particular presentation adds structure and discussion points to the session's overall objective of the tools and methodologies used by researchers in addressing concerns about the adequacy of sampled respondents in construct/scale measurement development, data quality, and representativeness to defined target populations. 